\title{
Modelling and optimisation of a traffic intersection based on queue theory and Markov decision control methods
}

\begin{abstract}
Traffic models play an important role in both today's traffic research and in many traffic applications such as traffic flow prediction, incident detection and traffic control. Modelling traffic dynamics and optimising the control signal are two interrelated problems. Modelling provides fundamental understanding of traffic dynamics and behaviour. In this paper, traffic signal is modelled as a M/M/1 queueing theory. The validation of a simulation model $(\mathrm{M} / \mathrm{M} / 1$ queue) with different arrival rates is presented. From the result, a traffic light model was developed by applying $\mathrm{M} / \mathrm{M} / 1$ queue theory for single intersection. In the optimisation strategy, the Markov decision control is applied to minimize queue length and waiting time. Simulation results show the excellent potential of this approach.
\end{abstract}

Keyword: Markov decision control; Queue theory; Traffic intersections; Traffic signals 